\title{
Generalized multiple Dirichlet series and generalized multiple polylogarithms
}

by

Kohji Matsumoto (Nagoya) and Hirofumi Tsumura (Tokyo)

1. Introduction and statement of results. Let $\mathbb{N}$ be the set of natural numbers, $\mathbb{N}_{0}=\mathbb{N} \cup\{0\}, \mathbb{Z}$ the ring of rational integers, $\mathbb{Q}$ the field of rational numbers, $\mathbb{R}$ the field of real numbers, and $\mathbb{C}$ the field of complex numbers.

Let $s=\sigma+i \tau$ be a complex variable, and

$$
\psi(s)=\sum_{n=0}^{\infty} \frac{a(n)}{(\beta+n w)^{s}}
$$

be a function with complex coefficients $a(n)$, where $\beta, w \in \mathbb{R}$ with $0<\beta \leq w$. We assume the following:

Assumption I. There exists a $q>0$ such that $\psi(s)$ is absolutely convergent for $\sigma>q$.

Throughout this paper we fix $\delta \in \mathbb{R}$ with $\delta>0$ and let $u \in \mathbb{R}$ with $1 \leq u \leq 1+\delta$. We let

$$
\psi(s ; u)=\sum_{n=0}^{\infty} \frac{a(n) u^{-n}}{(\beta+n w)^{s}} .
$$

By Assumption I, we can check that if $1<u \leq 1+\delta$ then the right-hand side of (1.2) is absolutely convergent for any $s \in \mathbb{C}$, so $\psi(s ; u)$ is holomorphic for all $s \in \mathbb{C}$. Corresponding to $\psi(s ; u)$, let

$$
G_{1}(t ; \psi ; u)=\sum_{n=0}^{\infty} a(n) u^{-n} e^{(\beta+n w) t},
$$

where $t$ is a complex variable. By Assumption I, the series (1.3) is convergent when $\Re t<0$. We further assume the following:

Assumption II. $\psi(s)$ can be continued analytically to the whole complex plane $\mathbb{C}$, and is holomorphic for all $s \in \mathbb{C}$. In any fixed strip $\sigma_{1} \leq \sigma \leq \sigma_{2}$,

2000 Mathematics Subject Classification: 11M41, 40B05. 
$\psi(s ; u)$ is uniformly convergent to $\psi(s)$ as $u \rightarrow 1+0$. Furthermore there exists a $\theta_{0}=\theta_{0}\left(\sigma_{1}, \sigma_{2}\right) \in \mathbb{R}$ with $0 \leq \theta_{0}<\pi / 2$ such that $\psi(s ; u)=O\left(e^{\theta_{0}|\tau|}\right)$ as $|\tau| \rightarrow \infty$.

Assumption III. There exists a $\varrho=\varrho(\psi)>0$ such that $G_{1}(t ; \psi ; u)$ can be continued holomorphically to

$$
\mathcal{D}(\varrho)=\{t \in \mathbb{C}|| t \mid<\varrho\}
$$

for any $u \in[1,1+\delta]$.

We will give typical examples which satisfy Assumptions I-III in Section 2 (see Example 2.2).

In the present paper, we consider generalized multiple Dirichlet series defined as follows. Let $\left(\alpha_{0}, \alpha_{1}, \ldots, \alpha_{r}\right) \in \mathbb{R}^{r+1}$ and $\left(w_{1}, \ldots, w_{r}\right) \in \mathbb{R}^{r}$ be such that $\alpha_{0}=0$ and $0<\alpha_{k}-\alpha_{k-1} \leq w_{k}(1 \leq k \leq r)$. Let $\mathcal{P}_{r}=\left\{\psi_{1}, \ldots, \psi_{r}\right\}$, where

$$
\psi_{k}(s)=\sum_{n=0}^{\infty} \frac{a_{k}(n)}{\left(\alpha_{k}-\alpha_{k-1}+n w_{k}\right)^{s}} .
$$

We assume that $\psi_{k}(s)$ and the associated series $\psi_{k}(s ; u), G_{1}\left(t ; \psi_{k} ; u\right)$ (defined similarly to (1.2) and (1.3)) satisfy Assumptions I-III $(1 \leq k \leq r)$. By Assumptions I and III, there exist $\left\{q_{k}=q\left(\psi_{k}\right)(>0) \mid 1 \leq k \leq r\right\}$ and $\left\{\varrho_{k}=\varrho\left(\psi_{k}\right)(>0) \mid 1 \leq k \leq r\right\}$. We let

$$
\eta_{r}=\min _{1 \leq k \leq r}\left\{\varrho_{k} / 2^{r-1}\right\} .
$$

We define the generalized multiple Dirichlet series associated with $\mathcal{P}_{r}$ by

$$
\Psi_{r}\left(s_{1}, \ldots, s_{r} ; u\right)=\sum_{n_{1}, \ldots, n_{r}=0}^{\infty} \frac{a_{1}\left(n_{1}\right) \cdots a_{r}\left(n_{r}\right) u^{-\sum_{\nu=1}^{r} n_{\nu}}}{\prod_{j=1}^{r}\left(\alpha_{j}+\sum_{\nu=1}^{j} n_{\nu} w_{\nu}\right)^{s_{j}}}
$$

for $s_{1}, \ldots, s_{r} \in \mathbb{C}$ and $u \in[1,1+\delta]$. The special case $u=1$ and $a_{j}(n)=1$ $(1 \leq j \leq r)$ has been studied by the first author in [12, 13]; it can be regarded as a generalization of both the Euler-Zagier multiple zeta function and the Barnes multiple zeta function. On the other hand, the special case $u=1, \alpha_{j}=j$ and $w_{j}=1(1 \leq j \leq r)$ has also been studied before: see Arakawa-Kaneko [2] when $a_{j}$ s are periodic functions on $\mathbb{Z}$, and MatsumotoTanigawa [14] for more general $a_{j}$ s.

First we prove the following result by using the method introduced by Matsumoto-Tanigawa [14] (see also [11-13]). Indeed, this can be regarded as a generalization of Theorem 2 in [14].

Theorem 1.1. For $s_{1}, \ldots, s_{r} \in \mathbb{C}$ and $u \in[1,1+\delta], \Psi_{r}\left(s_{1}, \ldots, s_{r} ; u\right)$ is absolutely convergent for $s_{j}=\sigma_{j}+i \tau_{j} \in \mathbb{C}(1 \leq j \leq r)$ with each $\sigma_{j}>q_{j}$. Furthermore $\Psi_{r}\left(s_{1}, \ldots, s_{r} ; u\right)$ can be continued analytically to the 
whole complex space $\mathbb{C}^{r}$ and is holomorphic on $\mathbb{C}^{r}$, and satisfies

$$
\lim _{u \rightarrow 1+0} \Psi_{r}\left(s_{1}, \ldots, s_{r} ; u\right)=\Psi_{r}\left(s_{1}, \ldots, s_{r} ; 1\right)
$$

for any $\left(s_{1}, \ldots, s_{r}\right) \in \mathbb{C}^{r}$.

REMARK 1.2. The meromorphic continuation of $\Psi_{r}\left(s_{1}, \ldots, s_{r} ; u\right)$ can be proved even if $\psi_{k}(s)$ has poles. When $u>1$, the multiple series (1.7) is absolutely convergent, hence holomorphic, for any $\left(s_{1}, \ldots, s_{r}\right) \in \mathbb{C}^{r}$. When $u=1$, if we assume that $\psi_{k}(s)$ has a pole of order at most one at $s=q_{k}$ and is holomorphic elsewhere (and satisfies $\psi_{k}(s)=O\left(e^{\theta_{0}|\tau|}\right)$ ) for $1 \leq k \leq r$, then we can show the following result, which generalizes Theorem 1 in [14]:

The function $\Psi_{r}\left(s_{1}, \ldots, s_{r} ; 1\right)$ can be continued meromorphically to the whole space $\mathbb{C}^{r}$, and its possible singularities are located only on the subsets of $\mathbb{C}^{r}$ defined by one of the following equations:

$$
\begin{aligned}
& s_{j}+\cdots+s_{r}=q_{j}+\delta_{j+1} q_{j+1}+\cdots+\delta_{r} q_{r}-n \\
& \left(1 \leq j \leq r, \delta_{k}=0 \text { or } 1(2 \leq k \leq r), n \in \mathbb{N}_{0}\right) .
\end{aligned}
$$

Moreover, (i) if $j=r \geq 2$ and $q_{r} \in \mathbb{N}$, then $n \leq q_{r}-1$, (ii) if $2 \leq j \leq r-1$, $q_{j} \in \mathbb{N}$ and $\delta_{j+1}=\cdots=\delta_{r}=1$, then $n \leq q_{r}-1$, (iii) if $j=r=1$ or if $j=1$ and $\delta_{2}=\cdots=\delta_{r}=1$, then $n=0$.

The proof uses the method of proof of Theorem 1 in [14].

We further consider generalized multiple polylogarithms related to (1.5). Let $\mathbf{d}_{r}=\left(d_{1}, \ldots, d_{r}\right) \in \mathbb{C}^{r}$ with $\Re d_{j}>q_{j}$ for each $j$. With the above notation, and for $u \in[1,1+\delta]$, let

$$
\begin{aligned}
& F_{r}\left(t_{1}, \ldots, t_{r} ; \mathbf{d}_{r} ; \mathcal{P}_{r} ; u\right) \\
& \quad=\sum_{n_{1}, \ldots, n_{r}=0}^{\infty} \frac{a_{1}\left(n_{1}\right) \cdots a_{r}\left(n_{r}\right) u^{-\sum_{l=1}^{r} n_{l}} \prod_{j=1}^{r} e^{\left(\alpha_{j}+\sum_{\mu=1}^{j} n_{\mu} w_{\mu}\right) t_{j}}}{\prod_{j=1}^{r}\left(\alpha_{j}+\sum_{\mu=1}^{j} n_{\mu} w_{\mu}\right)^{d_{j}}} .
\end{aligned}
$$

This multiple series is convergent when $\Re t_{j} \leq 0(1 \leq j \leq r)$. If we formally let $\psi_{k}(s)=\zeta(s)$, the Riemann zeta function, and $d_{k} \in \mathbb{N}$ $(1 \leq k \leq r)$ in $(1.9)$, then $F_{r}\left(\log x_{1}, \ldots, \log x_{r} ; \mathbf{d}_{r} ; \mathcal{P}_{r} ; 1\right)$ is the multiple polylogarithm defined by Goncharov [6] (see also [4]). However, $\zeta(s)$ does not satisfy Assumption II, so we will not consider the Goncharov multiple polylogarithms in this paper. Instead, we prove the following result.

TheOREM 1.3. For $\mathbf{d}_{r} \in \mathbb{C}^{r}$ with each $\Re d_{j}>q_{j}(1 \leq j \leq r)$ and $u \in$ $[1,1+\delta], F_{r}\left(t_{1}, \ldots, t_{r} ; \mathbf{d}_{r} ; \mathcal{P}_{r} ; u\right)$ is holomorphic for all $\left(t_{1}, \ldots, t_{r}\right) \in \mathcal{D}\left(\eta_{r}\right)^{r}$, 
and satisfies, for $\left(t_{1}, \ldots, t_{r}\right) \in \mathcal{D}\left(\eta_{r}\right)^{r}$,

$$
\begin{aligned}
F_{r}\left(t_{1}, \ldots, t_{r} ; \mathbf{d}_{r} ; \mathcal{P}_{r} ; u\right) & \\
= & \sum_{N_{1}, \ldots, N_{r}=0}^{\infty} \Psi_{r}\left(d_{1}-N_{1}, \ldots, d_{r}-N_{r} ; u\right) \frac{t_{1}^{N_{1}} \cdots t_{r}^{N_{r}}}{N_{1} ! \cdots N_{r} !} .
\end{aligned}
$$

Furthermore, for any $\xi \in \mathbb{R}$ with $0<\xi<\eta_{r}$, (1.10) is uniformly convergent with respect to $\left(t_{1}, \ldots, t_{r}, u\right) \in \overline{\mathcal{D}}(\xi)^{r} \times[1,1+\delta]$, where $\overline{\mathcal{D}}(\xi)=\{t \in \mathbb{C}|| t \mid \leq \xi\}$.

The special case $\psi_{j}(s)=\sum_{n \geq 1}(-1)^{n} n^{-s}(1 \leq j \leq r), \mathbf{d}_{r} \in \mathbb{N}^{r}$ and $t_{1}=\cdots=t_{r-1}=0$ has been studied by the second author. Indeed, $F_{r}\left(0, \ldots, 0, t ; \mathbf{d}_{r} ; \mathcal{P}_{r} ; u\right)$ played an important role in giving some evaluation formulas for Euler-Zagier sums (see [15]). In order to prove Theorem 1.3 and Proposition 2.1 (see below), we make use of the technique introduced in $[15]$.

As applications, using Theorem 1.3, we prove certain estimates for $\Psi_{r}\left(d_{1}-N_{1}, \ldots, d_{r}-N_{r} ; 1\right)$ (see Proposition 5.1 and Example 5.2). We further give certain multiple analogues of both Berndt's and Katsurada's formulas for Dirichlet $L$-functions proved in [3, 9] (see Example 5.3).

The authors wish to express their sincere gratitude to the referee for his (or her) valuable comments and important suggestions.

2. Generalized polylogarithms. First we consider the case of $r=1$. Let $\psi(s)$ be as defined in $(1.1)$ and $F_{1}(t ; d ; \psi ; u)$ as defined in (1.9). With the notation of Section 1, we can prove the following.

Proposition 2.1. For $d \in \mathbb{C}$ with $\Re d>q$ and $u \in[1,1+\delta], F_{1}(t ; d ; \psi ; u)$ is holomorphic for all $t \in \mathcal{D}(\varrho)$, and satisfies, for $t \in \mathcal{D}(\varrho)$,

$$
F_{1}(t ; d ; \psi ; u)=\sum_{N=0}^{\infty} \psi(d-N ; u) \frac{t^{N}}{N !} .
$$

Furthermore, for any $\xi \in \mathbb{R}$ with $0<\xi<\varrho,(2.1)$ is uniformly convergent with respect to $(t, u) \in \overline{\mathcal{D}}(\xi) \times[1,1+\delta]$.

Proof. By Assumption III, we can let

$$
G_{1}(t ; \psi ; u)=\sum_{n=0}^{\infty} \mathfrak{B}_{n}(\psi ; u) \frac{t^{n}}{n !}
$$

for $|t|<\varrho$. We use the method of contour integrals (see, for example, [16, proof of Theorem 4.2]). We consider the path $\Upsilon$ which consists of the positive real axis $[\varepsilon, \infty]$ (top side), a circle $C_{\varepsilon}$ around 0 of radius $\varepsilon$, and the positive real axis $[\varepsilon, \infty]$ (bottom side), where $0<\varepsilon<\varrho$. Note that we 
interpret $t^{s}$ to mean $\exp (s \log t)$, where the imaginary part of $\log t$ varies from 0 (on the top side of the real axis) to $2 \pi$ (on the bottom side). Let

$$
\begin{aligned}
& H_{1}(s ; \psi ; u)=\int_{\Upsilon} G_{1}(-t ; \psi ; u) t^{s-1} d t \\
& \quad=\left(e^{2 \pi i s}-1\right) \int_{\varepsilon}^{\infty} G_{1}(-t ; \psi ; u) t^{s-1} d t+\int_{C_{\varepsilon}} G_{1}(-t ; \psi ; u) t^{s-1} d t,
\end{aligned}
$$

which, in view of (1.3), is holomorphic for all $s \in \mathbb{C}$ if $0<\varepsilon<\varrho$. Putting $s=-n$ for $n \in \mathbb{N}_{0}$ and $\varepsilon=\xi$ with $0<\xi<\varrho$ in (2.3) and using (2.2), we have

$$
H_{1}(-n ; \psi ; u)=\int_{C_{\xi}} G_{1}(-t ; \psi ; u) t^{-n-1} d t=\frac{(2 \pi i) \mathfrak{B}_{n}(\psi ; u)(-1)^{n}}{n !}
$$

From Assumption III, $G_{1}(t ; \psi ; u)$ is continuous for all $(t, u) \in \mathcal{D}(\varrho) \times[1,1+\delta]$. Hence the value $\mathcal{M}_{\xi}=\max \left\{\left|G_{1}(-t ; \psi ; u)\right| \mid(t, u) \in\{t \in \mathbb{C}|| t \mid=\xi\} \times[1,1+\delta]\right\}$ exists. By the above equation, we have

$$
\frac{\left|\mathfrak{B}_{n}(\psi ; u)\right|}{n !} \leq \frac{1}{2 \pi} \int_{C_{\xi}}\left|G_{1}(-t ; \psi ; u)\right||t|^{-n-1}|d t| \leq \frac{\mathcal{M}_{\xi}}{\xi^{n}}
$$

for any $n \in \mathbb{N}_{0}$ and $u \in[1,1+\delta]$, where $\xi$ is an arbitrary real number with $0<\xi<\varrho$.

On the other hand, let $s \in \mathbb{C}$ with $\Re s>\max (1, q)$. Then the second term on the right-hand side of (2.3) tends to 0 as $\varepsilon \rightarrow 0$. Hence

$$
\begin{aligned}
H_{1}(s ; \psi ; u) & =\left(e^{2 \pi i s}-1\right) \int_{0}^{\infty} G_{1}(-t ; \psi ; u) t^{s-1} d t \\
& =\left(e^{2 \pi i s}-1\right) \sum_{n=0}^{\infty} a(n) u^{-n} \int_{0}^{\infty} t^{s-1} e^{-(\beta+n w) t} d t \\
& =\left(e^{2 \pi i s}-1\right) \Gamma(s) \psi(s ; u),
\end{aligned}
$$

where the interchange of summation and integration is valid because $\Re s>q$. Hence

$$
\psi(s ; u)=\frac{1}{\left(e^{2 \pi i s}-1\right) \Gamma(s)} H_{1}(s ; \psi ; u)=\frac{\Gamma(1-s)}{2 \pi i e^{\pi i s}} H_{1}(s ; \psi ; u),
$$

because

$$
\Gamma(s) \Gamma(1-s)=\frac{\pi}{\sin \pi s}=\frac{2 \pi i}{e^{\pi i s}-e^{-\pi i s}} .
$$

The relation (2.6) is valid for all $s \in \mathbb{C}$ by analytic continuation. 
Next, for $d \in \mathbb{C}$ with $\Re d>q$ and $N \in \mathbb{N}_{0}$, we put $s=d-N$ in (2.3). Then

$$
\begin{aligned}
H_{1}(d-N ; \psi ; u)= & \left(e^{2 \pi i d}-1\right) \int_{\varepsilon}^{\infty} G_{1}(-t ; \psi ; u) t^{d-N-1} d t \\
& +\int_{C_{\varepsilon}} G_{1}(-t ; \psi ; u) t^{d-N-1} d t=: I_{1}+I_{2} .
\end{aligned}
$$

Note that if $N \geq \Re d+1$ then

$$
\left|\int_{\varepsilon}^{\infty} e^{-(\beta+n w) t} t^{d-N-1} d t\right| \leq \frac{e^{-(\beta+n w) \varepsilon} \varepsilon^{\Re d-N-1}}{\beta+n w} .
$$

Hence

$$
\left|I_{1}\right| \leq \varepsilon^{\Re d-N-1}\left|e^{2 \pi i d}-1\right| \sum_{n=0}^{\infty} \frac{|a(n)| e^{-(\beta+n w) \varepsilon}}{\beta+n w} .
$$

On the other hand, by using the fact that

$$
\int_{C_{\varepsilon}} t^{p} d t= \begin{cases}2 \pi i & (p=-1), \\ \varepsilon^{p+1} \frac{e^{2 \pi i p}-1}{p+1} & (p \neq-1),\end{cases}
$$

for $p \in \mathbb{C}$ and by $(2.2)$, we have

$$
I_{2}= \begin{cases}(2 \pi i) \mathfrak{B}_{N-d}(\psi ; u) \frac{(-1)^{N-d}}{(N-d) !} & \left(N-d \in \mathbb{N}_{0}\right), \\ \varepsilon^{d-N}\left(e^{2 \pi i d}-1\right) \sum_{n=0}^{\infty} \frac{\mathfrak{B}_{n}(\psi ; u)(-1)^{n} \varepsilon^{n}}{(n+d-N) n !} & \text { (otherwise). }\end{cases}
$$

Note that the above infinite series is convergent because of the assumption $\varepsilon<\varrho$ and (2.4). Hence

$$
\left|I_{2}\right| \leq \begin{cases}2 \pi \frac{\left|\mathfrak{B}_{N-d}(\psi ; u)\right|}{(N-d) !} & \left(N-d \in \mathbb{N}_{0}\right), \\ \varepsilon^{\Re d-N}\left|e^{2 \pi i d}-1\right| \mid \sum_{n=0}^{\infty} \mathfrak{B}_{n}(\psi ; u) \frac{(-1)^{n} \varepsilon^{n}}{(n+d-N) n ! \mid} & \text { (otherwise). }\end{cases}
$$

From (2.4) with $\xi=\varepsilon$, the first case of (2.11) yields

$$
\left|I_{2}\right| \leq 2 \pi \mathcal{M}_{\varepsilon} \varepsilon^{d-N} \text {. }
$$

In the second case of (2.11), we let $\gamma_{d}=\min \{|d-m| \mid m \in \mathbb{Z}\}$. Using (2.4) with $\xi$ such that $0<\varepsilon<\xi<\varrho$, we see that the second case of (2.11) yields

$$
\left|I_{2}\right| \leq \varepsilon^{\Re d-N}\left|e^{2 \pi i d}-1\right| \frac{\mathcal{M}_{\xi}}{\gamma_{d}(1-\varepsilon / \xi)} .
$$


Hence it follows from (2.6)-(2.11) that there exists a constant $M>0$ which depends on $\varepsilon, d$ and $\psi$ but is independent of $N$ and $u$ such that

$$
\left|\frac{\psi(d-N ; u)}{\Gamma(1+N-d)}\right|=\frac{1}{2 \pi\left|e^{\pi i d}\right|}\left|H_{1}(d-N ; \psi ; u)\right| \leq M \varepsilon^{-N}
$$

for $N \in \mathbb{N}_{0}$ with $N \geq \Re d+1$. Note that we can take $\varepsilon$ arbitrary such that $0<\varepsilon<\varrho$. Since $|s| \leq|\Re s|+|\Im s|$ for $s \in \mathbb{C}$, we have

$$
\begin{aligned}
|\Gamma(1+N-d)| & =|(N-d)(N-d-1) \cdots([\Re d]+1-d) \Gamma([\Re d]+1-d)| \\
& \leq(N-[\Re d]+[|\Im d|]+1) !|\Gamma([\Re d]+1-d)|
\end{aligned}
$$

for $N \in \mathbb{N}_{0}$ with $N \geq \Re d+1$. Hence

$$
\begin{aligned}
& \frac{|\psi(d-N ; u)|}{N !} \\
& \leq \frac{(N-[\Re d]+[|\Im d|]+1) !|\Gamma([\Re d]+1-d)|}{N !}\left|\frac{\psi(d-N ; u)}{\Gamma(1+N-d)}\right| \\
& \quad \leq \frac{(N-[\Re d]+[|\Im d|]+1) !|\Gamma([\Re d]+1-d)|}{N !} M \varepsilon^{-N} .
\end{aligned}
$$

Suppose $u \in(1,1+\delta]$ and $t=i \theta$ with $\theta \in(-\varrho, \varrho) \subset \mathbb{R}$. Then there exists an $\varepsilon \in \mathbb{R}$ with $0<\varepsilon<\varrho$ and $|\theta|<\varepsilon$. From the definition (1.9) we have

$$
\begin{aligned}
F_{1}(i \theta ; d ; \psi ; u) & =\sum_{n=0}^{\infty} \frac{a(n) u^{-n}}{(\beta+n w)^{d}} \sum_{N=0}^{\infty} \frac{(\beta+n w)^{N}(i \theta)^{N}}{N !} \\
& =\sum_{N=0}^{\infty} \psi(d-N ; u) \frac{(i \theta)^{N}}{N !} .
\end{aligned}
$$

From (2.13) we can see that each side of (2.14) is uniformly convergent with respect to $u \in[1,1+\delta]$ because $|\theta|<\varepsilon$. Hence we can let $u \rightarrow 1$ on each side of $(2.14)$, so $(2.14)$ holds for $u=1$ when $\theta \in(-\varrho, \varrho)$. We can define

$$
F_{1}(t ; d ; \psi ; u)=\sum_{N=0}^{\infty} \psi(d-N ; u) \frac{t^{N}}{N !}
$$

for any $u \in[1,1+\delta]$ and $t \in \mathbb{C}$ with $|t|<\varrho$. From (2.13), this is uniformly convergent with respect to $(t, u) \in \overline{\mathcal{D}}(\xi) \times[1,1+\delta]$ when $0<\xi<\varrho$. Thus we have the assertion.

ExAMPLE 2.2. Let $f: \mathbb{Z} / m \mathbb{Z} \rightarrow \mathbb{C}$ be such that $\sum_{a=1}^{m} f(a)=0$. It can be regarded as a periodic function defined on $\mathbb{Z}$. For example, any non-trivial primitive Dirichlet character and any non-trivial additive character defined $\bmod m$ satisfy this condition. We define

$$
L(s ; f)=\sum_{n=1}^{\infty} \frac{f(n)}{n^{s}}
$$


and

$$
G_{1}(t ; L ; u)=\sum_{n=1}^{\infty} f(n) u^{-n} e^{n t}=\sum_{a=1}^{m} \frac{f(a) u^{-a} e^{a t}}{1-u^{-m} e^{m t}}
$$

for $u \in[1,1+\delta]$. Then $L(s ; f)$ and $G_{1}(t ; L ; u)$ satisfy Assumptions I-III. Note that $\varrho=2 \pi / m$ and $q=1$ in this case. For $d \in \mathbb{C}$ with $\Re d>1$, let

$$
F_{1}(t ; d ; L)=\sum_{n=1}^{\infty} \frac{f(n) e^{n t}}{n^{d}} .
$$

It follows from Proposition 2.1 that $F_{1}(t ; d ; L)$ is holomorphic on $\mathcal{D}(2 \pi / m)$ and satisfies

$$
F_{1}(t ; d ; L)=\sum_{N=0}^{\infty} L(d-N ; f) \frac{t^{N}}{N !} .
$$

In particular, when $f$ is a primitive Dirichlet character $\chi$ of conductor $m$, we know that $L(-2 j-1, \chi)=0$ if $\chi(-1)=-1$ and $L(-2 j, \chi)=0$ if $\chi(-1)=1$ for $j \in \mathbb{N}_{0}$ (see, for example, [16, Chap. 4]). Hence, applying (2.16) with $d=2 k$ and $d=2 k+1$ for $k \in \mathbb{N}$ and using $\cos x=\left(e^{i x}+e^{-i x}\right) / 2$, we obtain

$$
\begin{array}{ll}
\sum_{n=1}^{\infty} \frac{\chi(n) \cos (n \theta)}{n^{2 k}}=\sum_{j=0}^{k-1} L(2 k-2 j, \chi) \frac{(i \theta)^{2 j}}{(2 j) !} & (\chi(-1)=1), \\
\sum_{n=1}^{\infty} \frac{\chi(n) \cos (n \theta)}{n^{2 k+1}}=\sum_{j=0}^{k} L(2 k+1-2 j, \chi) \frac{(i \theta)^{2 j}}{(2 j) !} & (\chi(-1)=-1)
\end{array}
$$

for $\theta \in(-2 \pi / m, 2 \pi / m)$. These are typical examples of Berndt's result (see $[3$, Theorem 4.2]; see also [5, (1.2.12)]). Similarly, it follows from (2.16) that

$$
\begin{array}{ll}
\sum_{n=1}^{\infty} \frac{\chi(n) \cos (n \theta)}{n^{2 k+1}}=\sum_{j=0}^{\infty} L(2 k+1-2 j, \chi) \frac{(i \theta)^{2 j}}{(2 j) !} & (\chi(-1)=1), \\
\sum_{n=1}^{\infty} \frac{\chi(n) \cos (n \theta)}{n^{2 k}}=\sum_{j=0}^{\infty} L(2 k-2 j, \chi) \frac{(i \theta)^{2 j}}{(2 j) !} \quad(\chi(-1)=-1)
\end{array}
$$

for $k \in \mathbb{N}$ and $\theta \in(-2 \pi / m, 2 \pi / m)$. Using the functional equations for $L(s, \chi)$, we can confirm that these equations coincide with Katsurada's formulas for $L(s, \chi)$ (see $[9$, Theorem 3]).

3. Proof of Theorem 1.1. Using the method introduced in $[14$, Section 2] (see also [11-13]), we give the proof of Theorem 1.1 by induction on $r$. The case of $r=1$ can be directly obtained from Assumptions I and II. 
Hence we assume that Theorem 1.1 holds for $r-1$, and aim to prove the case of $r(\geq 2)$.

As in Section 1, let

$$
\Psi_{r}\left(s_{1}, \ldots, s_{r} ; u\right)=\Psi_{r}\left(s_{1}, \ldots, s_{r} ; \psi_{1}, \ldots, \psi_{r} ; u\right)
$$

be the function defined by (1.7). Since each $\psi_{k}(s)$ defined by $(1.5)$ converges absolutely for $\Re s>q_{k}(1 \leq k \leq r)$, we can easily check that $\Psi_{r}\left(s_{1}, \ldots, s_{r} ; u\right)$ converges absolutely if $\sigma_{k}=\Re s_{k}>q_{k}(1 \leq k \leq r)$.

First we assume each $\sigma_{k}>q_{k}(1 \leq k \leq r)$. Recall the Mellin-Barnes formula

$$
\Gamma(s)(1+\lambda)^{-s}=\frac{1}{2 \pi i} \int_{(c)} \Gamma(s+z) \Gamma(-z) \lambda^{z} d z,
$$

where $\Re s>0,|\arg \lambda|<\pi, \lambda \neq 0,-\Re s<c<0$, and the path of integration is the vertical line $\Re z=c$. By the above assumption, we may assume $-\sigma_{r}<$ $c<-q_{r}$. Put $s=s_{r}$ and

$$
\lambda=\frac{\alpha_{r}-\alpha_{r-1}+n_{r} w_{r}}{\alpha_{r-1}+n_{1} w_{1}+\cdots+n_{r-1} w_{r-1}}
$$

in (3.1). Then multiply both sides by

$$
\frac{a_{1}\left(n_{1}\right) \cdots a_{r}\left(n_{r}\right) u^{-\sum_{\nu=1}^{r} n_{\nu}}}{\prod_{j=1}^{r-2}\left(\alpha_{j}+\sum_{\nu=1}^{j} n_{\nu} w_{\nu}\right)^{s_{j}}\left(\alpha_{r-1}+\sum_{\nu=1}^{r-1} n_{\nu} w_{\nu}\right)^{s_{r-1}+s_{r}}}
$$

and sum up with respect to $n_{1}, \ldots, n_{r}$ to obtain

$$
\begin{aligned}
\Psi_{r}\left(s_{1}, \ldots, s_{r} ; u\right)= & \frac{1}{2 \pi i} \int_{(c)} \frac{\Gamma\left(s_{r}+z\right) \Gamma(-z)}{\Gamma\left(s_{r}\right)} \\
& \times \Psi_{r-1}\left(s_{1}, \ldots, s_{r-2}, s_{r-1}+s_{r}+z ; u\right) \psi_{r}(-z ; u) d z .
\end{aligned}
$$

Let $M \in \mathbb{N}$ and $\varepsilon \in \mathbb{R}$ be a small positive number. We shall shift the path to $\Re z=M-\varepsilon$. We see that

$$
\Psi_{r-1}\left(s_{1}, \ldots, s_{r-2}, s_{r-1}+s_{r}+z ; u\right)=O(1)
$$

in the region $c \leq \Re z \leq M-\varepsilon$ because $\sigma_{k}>q_{k}(1 \leq k \leq r-2),-\sigma_{r}<c$ and

$$
\sigma_{r-1}+\sigma_{r}+\Re z \geq \sigma_{r-1}+\sigma_{r}+c>\sigma_{r-1} .
$$

From the well-known Stirling formula for $\Gamma(s)$, we have

$$
|\Gamma(s)|=e^{-\pi|\tau| / 2}(|\tau|+1)^{\sigma-1 / 2}\left(1+O\left(\frac{1}{|\tau|+1}\right)\right)
$$

as $|\tau| \rightarrow \infty$, where $s=\sigma+i \tau$. Hence, by Assumption II, the integrand 
on the right-hand side of (3.2) tends to zero as $|\Im z| \rightarrow \infty$, so this shift is possible. By the inductive assumption, $\Psi_{r-1}$ is holomorphic on $\mathbb{C}^{r-1}$ and $\psi_{r}$ holomorphic on $\mathbb{C}$. Therefore we only have to count the residues of the poles of $\Gamma(-z)$ at $z=0,1, \ldots, M-1$. Since the residue of the pole of $\Gamma\left(s_{r}+z\right) \Gamma(-z) / \Gamma\left(s_{r}\right)$ at $z=k$ equals $-\left(\begin{array}{c}-s_{r} \\ k\end{array}\right)$, we obtain

$$
\begin{aligned}
& \Psi_{r}\left(s_{1}, \ldots, s_{r} ; u\right) \\
& =\sum_{k=0}^{M-1}\left(\begin{array}{c}
-s_{r} \\
k
\end{array}\right) \Psi_{r-1}\left(s_{1}, \ldots, s_{r-2}, s_{r-1}+s_{r}+k ; u\right) \psi_{r}(-k ; u) \\
& \quad+\frac{1}{2 \pi i} \int_{(M-\varepsilon)} \frac{\Gamma\left(s_{r}+z\right) \Gamma(-z)}{\Gamma\left(s_{r}\right)} \\
& \quad \times \Psi_{r-1}\left(s_{1}, \ldots, s_{r-2}, s_{r-1}+s_{r}+z ; u\right) \psi_{r}(-z ; u) d z=: S_{1}+S_{2} .
\end{aligned}
$$

Now $S_{1}$ is holomorphic on the whole $\mathbb{C}^{r}$ by the inductive assumption. On the other hand, $\Gamma\left(s_{r}+z\right)$ has no pole on the path $(M-\varepsilon)$, when $\Re\left(-s_{r}\right)=$ $-\sigma_{r}<M-\varepsilon$, so that $\sigma_{r}>-M+\varepsilon$. Using (3.3) and Assumption II, we see that $S_{2}$ is absolutely convergent, so it is holomorphic in the region

$$
\left\{\left(s_{1}, \ldots, s_{r}\right) \in \mathbb{C}^{r} \mid \sigma_{1}>q_{1}, \ldots, \sigma_{r-1}>q_{r-1}, \sigma_{r}>-M+\varepsilon\right\},
$$

where $M$ is arbitrary.

Next we fix $s_{r} \in \mathbb{C}$ with $\sigma_{r}>-M+\varepsilon$, and consider the continuation with respect to $s_{k}$ for $1 \leq k \leq r-1$. Since $\Psi_{r-1}$ is holomorphic on $\mathbb{C}^{r-1}$, the integrand in $S_{2}$ is holomorphic for all $\left(s_{1}, \ldots, s_{r-1}\right) \in \mathbb{C}^{r-1}$. So, if we prove that $S_{2}$ converges absolutely for any $\left(s_{1}, \ldots, s_{r-1}\right) \in \mathbb{C}^{r-1}$ and $s_{r} \in \mathbb{C}$ with $\sigma_{r}>-M+\varepsilon$, then $\Psi_{r}\left(s_{1}, \ldots, s_{r} ; u\right)$ is holomorphic on the whole $\mathbb{C}^{r}$ because $M$ is arbitrary. In order to prove this result, we need the following lemma.

Lemma 3.1. For $r \in \mathbb{N}$ with $r \geq 2$, there exists a polynomial $P_{r}(X) \in$ $\mathbb{R}[X]$ such that

$$
\Psi_{r}\left(s_{1}, \ldots, s_{r} ; u\right)=O\left(P_{r}\left(\left|\tau_{r}\right|\right) e^{\theta_{0}\left|\tau_{r}\right|}\right) \quad\left(\left|\tau_{r}\right| \rightarrow \infty\right)
$$

for any $\left(s_{1}, \ldots, s_{r-1}\right) \in \mathbb{C}^{r-1}$ and $u \in[1,1+d]$, where the constant implied by the $O$-symbol depends on $\tau_{1}, \ldots, \tau_{r-1}$.

Proof. We denote (3.5) by

$$
\Psi_{r}\left(s_{1}, \ldots, s_{r} ; u\right) \ll P_{r}\left(\left|\tau_{r}\right|\right) e^{\theta_{0}\left|\tau_{r}\right|} .
$$

We prove this lemma by induction on $r(\geq 2)$. First we consider the case of 
$r=2$. It follows from Assumption II and (3.4) that

$$
\begin{aligned}
& \left|\Psi_{2}\left(s_{1}, s_{2} ; u\right)\right| \\
& \leq \sum_{k=0}^{M-1}\left|\left(\begin{array}{c}
-s_{2} \\
k
\end{array}\right)\right|\left|\Psi_{1}\left(s_{1}+s_{2}+k ; u\right) \psi_{2}(-k ; u)\right| \\
& \quad+\frac{1}{2 \pi}\left|\int_{(M-\varepsilon)} \frac{\Gamma\left(s_{r}+z\right) \Gamma(-z)}{\Gamma\left(s_{r}\right)} \Psi_{1}\left(s_{1}+s_{2}+z ; u\right) \psi_{2}(-z ; u) d z\right| \\
& \ll \sum_{k=0}^{M-1}\left|\left(\begin{array}{c}
-s_{2} \\
k
\end{array}\right)\right| e^{\theta_{0}\left|\tau_{2}\right|}+\frac{1}{2 \pi} \int_{-\infty}^{\infty}\left|\frac{\Gamma\left(s_{r}+z\right) \Gamma(-z)}{\Gamma\left(s_{r}\right)}\right| e^{\theta_{0}\left|\tau_{2}+y\right|} e^{\theta_{0}|y|} d y,
\end{aligned}
$$

where $z=x+i y$. For simplicity, we denote the last term on the right-hand side of (3.6) by $I$. Using (3.3), we have

$$
\begin{aligned}
I \ll e^{\pi\left|\tau_{2}\right| / 2}\left(\left|\tau_{2}\right|+1\right)^{-\sigma_{2}+1 / 2} \\
\times \int_{-\infty}^{\infty} e^{\left(\theta_{0}-\pi / 2\right)\left|\tau_{2}+y\right|} e^{\left(\theta_{0}-\pi / 2\right)|y|}\left(\left|\tau_{2}+y\right|+1\right)^{\sigma_{2}+x-1 / 2}(|y|+1)^{-x-1 / 2} d y .
\end{aligned}
$$

Now Lemma 4 in [12] applied with $A=B=\theta_{0}-\pi / 2, p=\sigma_{2}+x-1 / 2$ and $q=-x-1 / 2$ yields

$$
\begin{aligned}
I \ll & e^{\pi\left|\tau_{2}\right| / 2}\left(\left|\tau_{2}\right|+1\right)^{-\sigma_{2}+1 / 2} \\
& \times\left[\left\{1+\left(\left|\tau_{2}\right|+1\right)^{\sigma_{2}+x-1 / 2}\right\}\left(\left|\tau_{2}\right|+1\right)^{-x+1 / 2} e^{\left(\theta_{0}-\pi / 2\right)\left|\tau_{2}\right|}\right. \\
& \left.+\left\{1+\left(\left|\tau_{2}\right|+1\right)^{\sigma_{2}+x-1 / 2}\right\} e^{\left(\theta_{0}-\pi / 2\right)\left|\tau_{2}\right|}\right] .
\end{aligned}
$$

Combining (3.6) and (3.8), we see that there exists $P_{2}(X) \in \mathbb{R}[X]$ such that

$$
\Psi_{2}\left(s_{1}, s_{2} ; u\right) \ll P_{2}\left(\left|\tau_{2}\right|\right) e^{\theta_{0}\left|\tau_{2}\right|} \quad\left(\left|\tau_{2}\right| \rightarrow \infty\right) .
$$

Thus we have the assertion for $r=2$.

Assume that the assertion holds for $r-1$. Substituting the assumed bounds into (3.4) and using Assumption II, we have

$$
\begin{aligned}
\Psi_{r}\left(s_{1}, \ldots, s_{r} ; u\right) \ll & \sum_{k=0}^{M-1}\left|\left(\begin{array}{c}
-\sigma_{r}+i \tau_{r} \\
k
\end{array}\right)\right| P_{r-1}\left(\left|\tau_{r-1}+\tau_{r}\right|\right) e^{\theta_{0}\left|\tau_{r-1}+\tau_{r}\right|} \\
& +\frac{1}{2 \pi i} \int_{-\infty}^{\infty}\left|\frac{\Gamma\left(s_{r}+z\right) \Gamma(-z)}{\Gamma\left(s_{r}\right)}\right| \\
& \times P_{r-1}\left(\left|\tau_{r-1}+\tau_{r}+y\right|\right) e^{\theta_{0}\left|\tau_{r-1}+\tau_{r}+y\right|} e^{\theta_{0}|y|} d y .
\end{aligned}
$$

By the same method as above, we can see that there exists $P_{r}(X) \in \mathbb{R}[X]$ such that

$$
\Psi_{r}\left(s_{1}, \ldots, s_{r} ; u\right) \ll P_{r}\left(\left|\tau_{r}\right|\right) e^{\theta_{0}\left|\tau_{r}\right|} .
$$

This finishes the proof of Lemma 3.1. 
Now we can complete the proof of Theorem 1.1 as follows. If we fix any $\left(s_{1}, \ldots, s_{r}\right) \in \mathbb{C}^{r}$, then it follows from Lemma 3.1 that

$$
\Psi_{r-1}\left(s_{1}, \ldots, s_{r-2}, s_{r-1}+s_{r}+z ; u\right) \ll P_{r-1}\left(\left|\tau_{r-1}+\tau_{r}+y\right|\right) e^{\theta_{0}\left|\tau_{r-1}+\tau_{r}+y\right|}
$$

as $|y| \rightarrow \infty$, where $z=x+i y$. Since $s_{r-1}$ is fixed, this can be written as

$$
\Psi_{r-1}\left(s_{1}, \ldots, s_{r-2}, s_{r-1}+s_{r}+z ; u\right) \ll \widetilde{P}_{r-1}\left(\left|\tau_{r}+y\right|\right) e^{\theta_{0}\left|\tau_{r}+y\right|} \quad(|y| \rightarrow \infty),
$$

where $\widetilde{P}_{r-1}(X) \in \mathbb{R}[X]$. Recall that $S_{2}$ is the second term on the right-hand side of (3.4). Then, by using (3.3), and by (3.8) and Assumption II, we have

$$
\begin{aligned}
S_{2} & \ll \int_{-\infty}^{\infty} \widetilde{\widetilde{P}}_{r-1}(y) e^{-\pi|y| / 2-\pi\left|\tau_{r}+y\right| / 2} e^{\theta_{0}\left|\tau_{r}+y\right|} e^{\theta_{0}|y|} d y \\
& =\int_{-\infty}^{\infty} \widetilde{\widetilde{P}}_{r-1}(y) e^{\left(\theta_{0}-\pi / 2\right)\left(\left|\tau_{r}+y\right|+|y|\right)} d y
\end{aligned}
$$

for some $\widetilde{\widetilde{P}}_{r-1}(X) \in \mathbb{R}[X]$. Since $0 \leq \theta_{0}<\pi / 2, S_{2}$ converges absolutely for any $\left(s_{1}, \ldots, s_{r}\right) \in \mathbb{C}^{r}$. By $(3.4), \Psi_{r}\left(s_{1}, \ldots, s_{r} ; u\right)$ is holomorphic on $\mathbb{C}^{r}$.

Lastly, we prove (1.8). More precisely, we prove that (1.8) holds uniformly with respect to $s_{j}(1 \leq j \leq r)$ in any fixed strip $\sigma_{1 j} \leq \Re s_{j} \leq \sigma_{2 j}$ as $u \rightarrow 1+0$. The case of $r=1$ follows from Assumption II. Hence we assume that the case of $r-1$ holds and prove the case of $r(\geq 2)$. Let $u \rightarrow 1+0$ in (3.4). From the inductive assumption, the integrand in $S_{2}$ is uniformly convergent with respect to $z$ in any fixed strip $\sigma_{1} \leq \Re z(=M-\varepsilon) \leq \sigma_{2}$ as $u \rightarrow 1+0$. Exchanging $\lim _{u \rightarrow 1+0}$ and the integral, and using the inductive assumption, we see that the right-hand side of (3.4) tends to

$$
\begin{aligned}
\sum_{k=0}^{M-1}\left(\begin{array}{c}
-s_{r} \\
k
\end{array}\right) \Psi_{r-1}\left(s_{1}, \ldots, s_{r-2}, s_{r-1}+s_{r}+k ; 1\right) \psi_{r}(-k ; 1) \\
+\frac{1}{2 \pi i} \int_{(M-\varepsilon)} \frac{\Gamma\left(s_{r}+z\right) \Gamma(-z)}{\Gamma\left(s_{r}\right)} \\
\times \Psi_{r-1}\left(s_{1}, \ldots, s_{r-2}, s_{r-1}+s_{r}+z ; 1\right) \psi_{r}(-z ; 1) d z
\end{aligned}
$$

as $u \rightarrow 1+0$. It is clear that this convergence is uniform with respect to $s_{j}$ in any fixed strip $\sigma_{1 j} \leq \Re s_{j} \leq \sigma_{2 j}(1 \leq j \leq r)$. From (3.4), we see that (3.10) coincides with $\Psi_{r}\left(s_{1}, \ldots, s_{r} ; 1\right)$. Hence the assertion in the case of $r$ holds. This completes the proof of Theorem 1.1.

Remark 3.2. For any $N \in \mathbb{N}_{0}$, let $M=N+1$ and $s_{r} \rightarrow-N$ in (3.4). Then $S_{2}$ tends to 0 because $\Gamma\left(s_{r}\right)$ has a pole at $s_{r}=-N$. Hence 
we obtain

$$
\begin{aligned}
& \Psi_{r}\left(s_{1}, \ldots, s_{r-1},-N ; u\right) \\
& \quad=\sum_{\nu=0}^{N}\left(\begin{array}{c}
N \\
\nu
\end{array}\right) \Psi_{r-1}\left(s_{1}, \ldots, s_{r-2}, s_{r-1}+\nu-N ; u\right) \psi_{r}(-\nu ; u)
\end{aligned}
$$

for $u \in[1,1+\delta]$ and $\left(s_{1}, \ldots, s_{r-1}\right) \in \mathbb{C}^{r-1}$. In particular, let $\psi_{j}(s)=L\left(s ; f_{j}\right)$ $(1 \leq j \leq r)$ and $u=1$, where each $f_{j}$ is defined $\bmod m_{j}$ and satisfies a certain condition (see Example 2.2). Then we can check that Assumptions I-III hold. In this case, $\Psi_{r}\left(s_{1}, \ldots, s_{r} ; 1\right)$ coincides with the multiple L-function

$$
L_{r}\left(s_{1}, \ldots, s_{r} ; f_{1}, \ldots, f_{r}\right)=\sum_{n_{1}, \ldots, n_{r}=1}^{\infty} \frac{f_{1}\left(n_{1}\right) \cdots f_{r}\left(n_{r}\right)}{n_{1}^{s_{1}}\left(n_{1}+n_{2}\right)^{s_{2}} \cdots\left(n_{1}+\cdots+n_{r}\right)^{s_{r}}},
$$

which has been studied in [2]. Hence (3.11) gives

$$
\begin{aligned}
& L_{r}\left(s_{1}, \ldots, s_{r-1},-N ; f_{1}, \ldots, f_{r}\right) \\
= & \sum_{\nu=0}^{N}\left(\begin{array}{c}
N \\
\nu
\end{array}\right) L_{r-1}\left(s_{1}, \ldots, s_{r-2}, s_{r-1}+\nu-N ; f_{1}, \ldots, f_{r-1}\right) L_{1}\left(-\nu ; f_{r}\right)
\end{aligned}
$$

for $\left(s_{1}, \ldots, s_{r-1}\right) \in \mathbb{C}^{r-1}$. This result was proved by Kamano (see [8]) by using the method introduced in [1]. This case can also be derived directly from the relation $(2.3)$ in [14].

4. Proof of Theorem 1.3. In this section, we prove Theorem 1.3 by induction on $r$.

The case of $r=1$ is just what we proved in Proposition 2.1. Hence we assume that the assertion holds for $r-1$ and prove the case of $r(\geq 2)$.

Let $\mathcal{P}_{r}=\left\{\psi_{1}, \ldots, \psi_{r}\right\}$ satisfy Assumptions I-III. Then we can take $\left\{q_{k}\right\}_{1 \leq k \leq r}$ and $\left\{\varrho_{k}\right\}_{1 \leq k \leq r}$, and define $\eta_{r-1}$ and $\eta_{r}$ by (1.6). Let

$$
\begin{aligned}
& G_{r}\left(t_{1}, \ldots, t_{r} ; \mathbf{d}_{r-1} ; \mathcal{P}_{r} ; u\right) \\
& \quad=F_{r-1}\left(t_{1}, \ldots, t_{r-2}, t_{r-1}+t_{r} ; \mathbf{d}_{r-1} ; \mathcal{P}_{r-1} ; u\right) G_{1}\left(t_{r} ; \psi_{r} ; u\right) \\
& =\sum_{n_{1}, \ldots, n_{r}=0}^{\infty} \frac{a_{1}\left(n_{1}\right) \cdots a_{r}\left(n_{r}\right) u^{-\sum_{l=1}^{r} n_{l}} \prod_{j=1}^{r} e^{\left(\alpha_{j}+\sum_{\mu=1}^{j} n_{\mu} w_{\mu}\right) t_{j}}}{\prod_{j=1}^{r-1}\left(\alpha_{j}+\sum_{\mu=1}^{j} n_{\mu} w_{\mu}\right)^{d_{j}}},
\end{aligned}
$$

which is convergent when $\Re t_{j}<0(1 \leq j \leq r)$. By the inductive assumption, $F_{r-1}\left(t_{1}, \ldots, t_{r-1}+t_{r} ; \mathbf{d}_{r-1} ; \mathcal{P}_{r-1} ; u\right)$ is holomorphic for $\left(t_{1}, \ldots, t_{r}\right) \in$ $\mathcal{D}\left(\eta_{r-1}\right)^{r-2} \times \mathcal{D}\left(\eta_{r-1} / 2\right)^{2}$, and $G_{1}\left(t_{r} ; \psi_{r} ; u\right)$ is holomorphic for $t_{r} \in \mathcal{D}\left(\varrho_{r}\right)$. Since $\eta_{r} \leq \min \left(\eta_{r-1} / 2, \varrho_{r}\right)$, we see that $G_{r}\left(t_{1}, \ldots, t_{r} ; \mathbf{d}_{r-1} ; \mathcal{P}_{r} ; u\right)$ is holomorphic for $\left(t_{1}, \ldots, t_{r}\right) \in \mathcal{D}\left(\eta_{r}\right)^{r}$. Therefore, if we fix $t_{r} \in \mathcal{D}\left(\eta_{r}\right)$ then the function of $r-1$ real variables $G_{r}\left(i \theta_{1}, \ldots, i \theta_{r-1}, t_{r} ; \mathbf{d}_{r-1} ; \mathcal{P}_{r} ; u\right)$ is real- 
analytic for $\left(\theta_{1}, \ldots, \theta_{r-1}\right) \in\left(-\eta_{r}, \eta_{r}\right)^{r-1} \subset \mathbb{R}^{r-1}$ (see, for example, [10, Corollary 2.3.7]). Similarly, if we fix $\left(\theta_{1}, \ldots, \theta_{r-1}\right) \in\left(-\eta_{r}, \eta_{r}\right)^{r-1}$, then $G_{r}\left(\left\{i \theta_{k}\right\}, t_{r} ; \mathbf{d}_{r-1} ; \mathcal{P}_{r} ; u\right)$ is holomorphic for $t_{r} \in \mathcal{D}\left(\eta_{r}\right)$. Hence we define $\left\{\mathfrak{B}_{n}\left(\left\{i \theta_{k}\right\} ; \mathbf{d}_{r-1} ; \mathcal{P}_{r} ; u\right)\right\}_{n \geq 0}$ by

$$
G_{r}\left(i \theta_{1}, \ldots, i \theta_{r-1}, t_{r} ; \mathbf{d}_{r-1} ; \mathcal{P}_{r} ; u\right)=\sum_{n=0}^{\infty} \mathfrak{B}_{n}\left(\left\{i \theta_{k}\right\} ; \mathbf{d}_{r-1} ; \mathcal{P}_{r} ; u\right) \frac{t_{r}^{n}}{n !} .
$$

As in the proof of Proposition 2.1, we let

$$
\begin{aligned}
& H_{r}\left(s ; i \theta_{1}, \ldots, i \theta_{r-1} ; \mathbf{d}_{r-1} ; \mathcal{P}_{r} ; u\right) \\
& =\int_{\Upsilon} G_{r}\left(\left\{i \theta_{k}\right\},-t ; \mathbf{d}_{r-1} ; \mathcal{P}_{r} ; u\right) t^{s-1} d t \\
& =\left(e^{2 \pi i s}-1\right) \int_{\varepsilon}^{\infty} G_{r}\left(\left\{i \theta_{k}\right\},-t ; \mathbf{d}_{r-1} ; \mathcal{P}_{r} ; u\right) t^{s-1} d t \\
& \quad+\int_{C_{\varepsilon}} G_{r}\left(\left\{i \theta_{k}\right\},-t ; \mathbf{d}_{r-1} ; \mathcal{P}_{r} ; u\right) t^{s-1} d t,
\end{aligned}
$$

which is holomorphic for all $s \in \mathbb{C}$ if we fix $\left(\theta_{1}, \ldots, \theta_{r-1}\right) \in\left(-\eta_{r}, \eta_{r}\right)^{r-1}$ and $0<\varepsilon<\eta_{r}$.

Putting $s=-n$ for $n \in \mathbb{N}_{0}$ and $\varepsilon=\xi$ with $0<\xi<\eta_{r}$ in (4.3), and using (4.2), we have

$$
\begin{aligned}
H_{r}\left(-n ; i \theta_{1}, \ldots, i \theta_{r-1} ; \mathbf{d}_{r-1} ; \mathcal{P}_{r} ; u\right) & =\int_{C_{\xi}} G_{r}\left(\left\{i \theta_{k}\right\},-t ; \mathbf{d}_{r-1} ; \mathcal{P}_{r} ; u\right) t^{-n-1} d t \\
& =\frac{(2 \pi i) \mathfrak{B}_{n}\left(\left\{i \theta_{k}\right\} ; \mathbf{d}_{r-1} ; \mathcal{P}_{r} ; u\right)(-1)^{n}}{n !} .
\end{aligned}
$$

By the inductive assumption and (4.1), we see that the Taylor expansion of $G_{r}\left(\left\{i \theta_{k}\right\},-t ; \mathbf{d}_{r-1} ; \mathcal{P}_{r} ; u\right)$ around $t=0$ is uniformly convergent with respect to $\left(\theta_{1}, \ldots, \theta_{r-1}, t, u\right) \in[-\xi, \xi]^{r-1} \times \overline{\mathcal{D}}(\xi) \times[1,1+\delta]$ when $\xi \in \mathbb{R}$ with $0<\xi<\eta_{r}$. In particular, $G_{r}\left(\left\{i \theta_{k}\right\},-t ; \mathbf{d}_{r-1} ; \mathcal{P}_{r} ; u\right)$ is continuous for $\left(\theta_{1}, \ldots, \theta_{r-1}, t, u\right) \in[-\xi, \xi]^{r-1} \times \overline{\mathcal{D}}(\xi) \times[1,1+\delta]$. Hence the value

$$
\begin{aligned}
\tilde{\mathcal{M}}_{\xi}=\max \left\{\mid G_{r}\left(\left\{i \theta_{k}\right\},-t ;\right.\right. & \left.\mathbf{d}_{r-1} ; \mathcal{P}_{r} ; u\right)|| \\
& \left.(t, u) \in[-\xi, \xi]^{r-1} \times\{|t|=\xi\} \times[1,1+\delta]\right\}
\end{aligned}
$$

exists when $\xi \in \mathbb{R}$ with $0<\xi<\eta_{r}$. By the above equation, we have

$$
\frac{\left|\mathfrak{B}_{n}\left(\left\{i \theta_{k}\right\} ; \mathbf{d}_{r-1} ; \mathcal{P}_{r} ; u\right)\right|}{n !} \leq \frac{\widetilde{\mathcal{N}}_{\xi}}{\xi^{n}}
$$

for any $n \in \mathbb{N}_{0},\left(\theta_{1}, \ldots, \theta_{r-1}\right) \in[-\xi, \xi]^{r-1}$ and $u \in[1,1+\delta]$. 


\section{Define}

$$
\begin{array}{r}
\mathcal{Z}_{r}\left(\mathbf{d}_{r-1}, s ; i \theta_{1}, \ldots, i \theta_{r-1} ; \mathcal{P}_{r} ; u\right)=F_{r}\left(i \theta_{1}, \ldots, i \theta_{r-1}, 0 ; \mathbf{d}_{r-1}, s ; \mathcal{P}_{r} ; u\right) \\
=\sum_{n_{1}, \ldots, n_{r}=0}^{\infty} \frac{a_{1}\left(n_{1}\right) \cdots a_{r}\left(n_{r}\right) u^{-\sum_{\nu=1}^{r} n_{\nu}} \prod_{j=1}^{r-1} e^{\left(\alpha_{j}+\sum_{\mu=1}^{j} n_{\mu} w_{\mu}\right) i \theta_{j}}}{\prod_{j=1}^{r-1}\left(\alpha_{j}+\sum_{\nu=1}^{j} n_{\nu} w_{\nu}\right)^{d_{j}}\left(\alpha_{r}+\sum_{\nu=1}^{r} n_{\nu} w_{\nu}\right)^{s}}
\end{array}
$$

for $\left(\theta_{1}, \ldots, \theta_{r-1}\right) \in\left(-\eta_{r}, \eta_{r}\right)^{r-1}, s \in \mathbb{C}$ with $\Re s>q_{r}$ and $u \in[1,1+\delta]$. Assuming $\Re s>\max \left(1, q_{r}\right)$ and using the same method as in the proof of Proposition 2.1, we have

$$
\begin{aligned}
Z_{r}\left(\mathbf{d}_{r-1}, s ;\left\{i \theta_{k}\right\} ; \mathcal{P}_{r} ; u\right) & =\frac{1}{\left(e^{2 \pi i s}-1\right) \Gamma(s)} H_{r}\left(s ;\left\{i \theta_{k}\right\} ; \mathbf{d}_{r-1} ; \mathcal{P}_{r} ; u\right) \\
& =\frac{\Gamma(1-s)}{2 \pi i e^{\pi i s}} H_{r}\left(s ;\left\{i \theta_{k}\right\} ; \mathbf{d}_{r-1} ; \mathcal{P}_{r} ; u\right)
\end{aligned}
$$

Note that $H_{r}\left(s ;\left\{i \theta_{k}\right\} ; \mathbf{d}_{r-1} ; \mathcal{P}_{r} ; u\right)$ is holomorphic for all $s \in \mathbb{C}$ if we fix $\left\{\theta_{k}\right\} \in\left(-\eta_{r}, \eta_{r}\right)^{r-1}$ (as mentioned above), and the poles of $\Gamma(1-s)$ coincide with $\mathbb{N}=\{1,2, \ldots\}$. Since $\mathcal{Z}_{r}\left(\mathbf{d}_{r-1}, s ;\left\{i \theta_{k}\right\} ; \mathcal{P}_{r} ; u\right)$ is absolutely convergent for $s \in \mathbb{C}$ with $\Re s>q_{r}$, it follows from (4.6) that $\mathcal{Z}_{r}\left(\mathbf{d}_{r-1}, s ;\left\{i \theta_{k}\right\} ; \mathcal{P}_{r} ; u\right)$ is defined and holomorphic for all $s \in \mathbb{C} \backslash\left\{1,2, \ldots,\left[q_{r}\right]\right\}$ if we fix $\left\{\theta_{k}\right\} \in$ $\left(-\eta_{r}, \eta_{r}\right)^{r-1}$.

Furthermore, we can prove that $\mathcal{Z}_{r}\left(\mathbf{d}_{r-1}, s ;\left\{i \theta_{k}\right\} ; \mathcal{P}_{r} ; u\right)$ has no pole as follows. Fix $s \in \mathbb{C}$. If $1<u \leq 1+\delta$ then from (1.7) and (4.5), and by substituting the Taylor expansion for each $\exp \left(\left(\alpha_{j}+\sum_{\nu=1}^{j} n_{\nu} w_{\nu}\right) i \theta_{j}\right)$ and changing the order of summations, we have

$$
\begin{aligned}
& z_{r}\left(\mathbf{d}_{r-1}, s ;\left\{i \theta_{k}\right\} ; \mathcal{P}_{r} ; u\right) \\
= & \sum_{N_{1}, \ldots, N_{r-1}=0}^{\infty} \Psi_{r}\left(d_{1}-N_{1}, \ldots, d_{r-1}-N_{r-1}, s ; u\right) \frac{\left(i \theta_{1}\right)^{N_{1}} \cdots\left(i \theta_{r-1}\right)^{N_{r-1}}}{N_{1} ! \cdots N_{r-1} !} .
\end{aligned}
$$

We see that (4.3) is uniformly convergent with respect to $\left(\theta_{1}, \ldots, \theta_{r-1}, u\right) \in$ $[-\xi, \xi]^{r-1} \times[1,1+\delta]$, for any $\xi \in \mathbb{R}$ with $0<\xi<\eta_{r}$. Hence, for $u \in[1,1+\delta]$, $H_{r}\left(s ;\left\{i \theta_{k}\right\} ; \mathbf{d}_{r-1} ; \mathcal{P}_{r} ; u\right)$ is real-analytic for $\left(\theta_{1}, \ldots, \theta_{r-1}\right) \in\left(-\eta_{r}, \eta_{r}\right)^{r-1}$. Put $\theta_{1}=\cdots=\theta_{r-1}=\theta$. Then for $u \in[1,1+\delta], H_{r}\left(s ;\{i \theta\} ; \mathbf{d}_{r-1} ; \mathcal{P}_{r} ; u\right)$ is realanalytic for $\theta \in\left(-\eta_{r}, \eta_{r}\right)$, and its Taylor expansion around $\theta=0$ is uniformly convergent with respect to $(\theta, u) \in[-\xi, \xi] \times[1,1+\delta]$. It follows from (4.6) that $Z_{r}\left(\mathbf{d}_{r-1}, s ;\{i \theta\} ; \mathcal{P}_{r} ; u\right)$ also has these properties. Hence, for any $u \in$ $[1,1+\delta]$, we define the one-variable complex function $z_{r}\left(\mathbf{d}_{r-1}, s ;\{t\} ; \mathcal{P}_{r} ; u\right)$ which is holomorphic for $t \in \mathcal{D}\left(\eta_{r}\right)$ and its Taylor expansion around $t=0$ is uniformly convergent with respect to $(t, u) \in \overline{\mathcal{D}}(\xi) \times[1,1+\delta]$. In particular, $\mathcal{Z}_{r}\left(\mathbf{d}_{r-1}, s ;\{t\} ; \mathcal{P}_{r} ; u\right)$ is continuous for $(t, u) \in \overline{\mathcal{D}}(\xi) \times[1,1+\delta]$. Putting $\xi=\varepsilon$ with $0<\varepsilon<\eta_{r}$ shows the existence of

$$
\mathcal{M}_{\varepsilon}^{\prime}=\max \left\{\left|Z_{r}\left(\mathbf{d}_{r-1}, s ;\{t\} ; \mathcal{P}_{r} ; u\right)\right| \mid(t, u) \in\{t \in \mathbb{C}|| t \mid=\varepsilon\} \times[1,1+\delta]\right\} .
$$


Using the same method as in the proof of (4.4) and by (4.7) and the continuity of $\Psi_{r}\left(d_{1}-N_{1}, \ldots, d_{r-1}-N_{r-1}, s ; u\right)$ in $u \in[1,1+\delta]$ (see Theorem 1.1), we see that

$$
\left|\sum_{N_{1}+\cdots+N_{r-1}=n} \frac{\Psi_{r}\left(d_{1}-N_{1}, \ldots, d_{r-1}-N_{r-1}, s ; u\right)}{N_{1} ! \cdots N_{r-1} !}\right| \leq \frac{\mathcal{M}_{\varepsilon}^{\prime}}{\varepsilon^{n}}
$$

for $u \in[1,1+\delta]$ and $n \in \mathbb{N}_{0}$, where $\varepsilon$ is an arbitrary real number with $0<\varepsilon<\eta_{r}$. This means that the right-hand side of (4.7) is uniformly convergent with respect to $\left(\theta_{1}, \ldots, \theta_{r-1}, u\right) \in[-\xi, \xi]^{r-1} \times[1,1+\delta]$ for any $\xi \in \mathbb{R}$ with $0<\xi<\eta_{r}$. Hence we can let $u \rightarrow 1$ in (4.7), so (4.7) holds for $u \in[1,1+\delta]$. Since $s$ is an arbitrary complex number, $\mathcal{Z}_{r}\left(\mathbf{d}_{r-1}, s ;\left\{i \theta_{k}\right\} ; \mathcal{P}_{r} ; u\right)$ has no pole, so it is holomorphic for all $s \in \mathbb{C}$ when $u \in[1,1+\delta]$, and real-analytic for $\left(\theta_{1}, \ldots, \theta_{r-1}\right) \in\left(-\eta_{r}, \eta_{r}\right)^{r-1}$ when $s \in \mathbb{C}$ and $u \in$ $[1,1+\delta]$

For $d_{r} \in \mathbb{C}$ with $\Re d_{r}>q_{r}$ and $N \in \mathbb{N}_{0}$ with $N \geq \Re d_{r}+1$, we put $s=d_{r}-N$ in (4.3). Then we have

$$
\begin{aligned}
H_{r}\left(d_{r}-\right. & \left.N ;\left\{i \theta_{k}\right\} ; \mathbf{d}_{r-1} ; \mathcal{P}_{r} ; u\right) \\
= & \left(e^{2 \pi i d_{r}}-1\right) \int_{\varepsilon}^{\infty} G_{r}\left(\left\{i \theta_{k}\right\},-t ; \mathbf{d}_{r-1} ; \mathcal{P}_{r} ; u\right) t^{d_{r}-N-1} d t \\
& \quad+\int_{C_{\varepsilon}} G_{r}\left(\left\{i \theta_{k}\right\},-t ; \mathbf{d}_{r-1} ; \mathcal{P}_{r} ; u\right) t^{d_{r}-N-1} d t=: J_{1}+J_{2} .
\end{aligned}
$$

Since $N \geq \Re d_{r}+1$, we have

$$
\left|\int_{\varepsilon}^{\infty} e^{-\left(\alpha_{r}+\sum_{\mu=1}^{r} n_{\mu} w_{\mu}\right) t} t^{d_{r}-N-1} d t\right| \leq \frac{e^{-\left(\alpha_{r}+\sum_{\mu=1}^{r} n_{\mu} w_{\mu}\right) \varepsilon}\left|\varepsilon^{d_{r}-N-1}\right|}{\alpha_{r}+\sum_{\mu=1}^{r} n_{\mu} w_{\mu}} .
$$

Hence

$$
\begin{aligned}
&\left|J_{1}\right| \leq \varepsilon^{\Re d_{r}-N-1}\left|e^{2 \pi i d_{r}}-1\right| \\
& \quad \times \sum_{n_{1}, \ldots, n_{r}=0}^{\infty} \frac{\left|a_{1}\left(n_{1}\right) \cdots a_{r}\left(n_{r}\right)\right| e^{-\left(\alpha_{r}+\sum_{\mu=1}^{r} n_{\mu} w_{\mu}\right) \varepsilon}}{\prod_{j=1}^{r-1}\left(\alpha_{j}+\sum_{\mu=1}^{j} n_{\mu} w_{\mu}\right)^{\Re d_{j}}\left(\alpha_{r}+\sum_{\mu=1}^{r} n_{\mu} w_{\mu}\right)} .
\end{aligned}
$$

On the other hand, by using (2.9), we have

$$
J_{2}= \begin{cases}(2 \pi i) \mathfrak{B}_{N-d_{r}}\left(\left\{i \theta_{k}\right\} ; \mathbf{d}_{r-1} ; \mathcal{P}_{r} ; u\right) \frac{(-1)^{N-d_{r}}}{\left(N-d_{r}\right) !} & \left(N-d_{r} \in \mathbb{N}_{0}\right), \\ \varepsilon^{d_{r}-N}\left(e^{2 \pi i d_{r}}-1\right) \sum_{n=0}^{\infty} \frac{\mathfrak{B}_{n}\left(\left\{i \theta_{k}\right\} ; \mathbf{d}_{r-1} ; \mathcal{P}_{r} ; u\right)(-1)^{n} \varepsilon^{n}}{\left(n+d_{r}-N\right) n !} & \text { (otherwise) } .\end{cases}
$$

The last series is uniformly convergent with respect to $\left(\theta_{1}, \ldots, \theta_{r-1}, u\right) \in$ 
$[-\varepsilon, \varepsilon]^{r-1} \times[1,1+\delta]$ because of the assumption $\varepsilon<\eta_{r}$ and (4.4). Hence either

$$
\left|J_{2}\right| \leq 2 \pi \frac{\left|\mathfrak{B}_{N-d_{r}}\left(\left\{i \theta_{k}\right\} ; \mathbf{d}_{r-1} ; \mathcal{P}_{r} ; u\right)\right|}{\left(N-d_{r}\right) !} \quad\left(N-d_{r} \in \mathbb{N}_{0}\right)
$$

or

$$
\left|J_{2}\right| \leq \varepsilon^{\Re d_{r}-N}\left|e^{2 \pi i d_{r}}-1\right|\left|\sum_{n=0}^{\infty} \mathfrak{B}_{n}\left(\left\{i \theta_{k}\right\} ; \mathbf{d}_{r-1} ; \mathcal{P}_{r} ; u\right) \frac{(-1)^{n} \varepsilon^{n}}{\left(n+d_{r}-N\right) n !}\right|
$$

(otherwise).

Just as (2.12), it follows from (4.4), (4.6), (4.8)-(4.12) that there exists a constant $M>0$ independent of $N$ and $\left\{\theta_{k}\right\}$ such that

$$
\begin{aligned}
& \left|\frac{z_{r}\left(\mathbf{d}_{r-1}, d_{r}-N ;\left\{i \theta_{k}\right\} ; \mathcal{P}_{r} ; u\right)}{\Gamma\left(1+N-d_{r}\right)}\right| \\
& \quad \leq \frac{1}{2 \pi \mid e^{\pi i d_{r} \mid}}\left|H_{r}\left(d_{r}-N ;\left\{i \theta_{k}\right\} ; \mathbf{d}_{r-1} ; \mathcal{P}_{r} ; u\right)\right| \leq M \varepsilon^{-N}
\end{aligned}
$$

for $N \in \mathbb{N}$ with $N \geq \Re d_{r}+1$. Note that we can take $\varepsilon$ arbitrary such that $0<\varepsilon<\eta_{r}$. As in the case of (2.13), we have

$$
\begin{aligned}
& \frac{\left|Z_{r}\left(\mathbf{d}_{r-1}, d_{r}-N ;\left\{i \theta_{k}\right\} ; \mathcal{P}_{r} ; u\right)\right|}{N !} \\
& \quad \leq \frac{\left(N-\left[\Re d_{r}\right]+\left[\left|\Im d_{r}\right|\right]+1\right) !\left|\Gamma\left(\left[\Re d_{r}\right]+1-d_{r}\right)\right|}{N !} M \varepsilon^{-N}
\end{aligned}
$$

for $N \in \mathbb{N}$ with $N \geq \Re d_{r}+1$ and $u \in[1,1+\delta]$.

Suppose $1<u \leq 1+\delta$ and $\theta_{r} \in\left(-\eta_{r}, \eta_{r}\right)$. Then by (1.9), and using the Taylor expansion for $\exp \left(\left(\alpha_{r}+\sum_{\nu=1}^{r} n_{\nu} w_{\nu}\right) i \theta_{r}\right)$, we have

$$
\begin{aligned}
& F_{r}\left(i \theta_{1}, \ldots, i \theta_{r-1}, i \theta_{r} ; \mathbf{d}_{r} ; \mathcal{P}_{r} ; u\right) \\
& =\sum_{N_{r}=0}^{\infty} z_{r}\left(\mathbf{d}_{r-1}, d_{r}-N_{r} ;\left\{i \theta_{k}\right\} ; \mathcal{P}_{r} ; u\right) \frac{\left(i \theta_{r}\right)^{N_{r}}}{N_{r} !} .
\end{aligned}
$$

By (4.14), the right-hand side of (4.15) is uniformly convergent with respect to $\left(\theta_{r}, u\right) \in[-\xi, \xi] \times[1,1+\delta]$ when $\left(\theta_{1}, \ldots, \theta_{r-1}\right) \in\left(-\eta_{r}, \eta_{r}\right)^{r-1}$ and $0<$ $\xi<\eta_{r}$. Hence (4.15) holds for $u=1$. As mentioned above, (4.7) holds for any $s \in \mathbb{C},\left(\theta_{1}, \ldots, \theta_{r-1}\right) \in\left(-\eta_{r}, \eta_{r}\right)^{r-1}$, and $u \in[1,1+\delta]$. Consequently

$$
\begin{aligned}
Z_{r}\left(\mathbf{d}_{r-1}, d_{r}-N_{r} ;\left\{i \theta_{k}\right\} ; \mathcal{P}_{r} ; u\right) \\
=\sum_{N_{1}, \ldots, N_{r-1}=0}^{\infty} \Psi_{r}\left(d_{1}-N_{1}, \ldots, d_{r}-N_{r} ; u\right) \frac{\left(i \theta_{1}\right)^{N_{1}} \cdots\left(i \theta_{r-1}\right)^{N_{r-1}}}{N_{1} ! \cdots N_{r-1} !}
\end{aligned}
$$


for $u \in[1,1+\delta]$. Hence (4.15) can also be written as

$$
\begin{aligned}
& F_{r}\left(i \theta_{1}, \ldots, i \theta_{r} ; \mathbf{d}_{r} ; \mathcal{P}_{r} ; u\right) \\
& \quad=\sum_{N_{1}, \ldots, N_{r}=0}^{\infty} \Psi_{r}\left(d_{1}-N_{1}, \ldots, d_{r}-N_{r} ; u\right) \frac{\left(i \theta_{1}\right)^{N_{1}} \cdots\left(i \theta_{r}\right)^{N_{r}}}{N_{1} ! \cdots N_{r} !}
\end{aligned}
$$

for $u \in[1,1+\delta]$, and (4.17) is uniformly convergent with respect to $\left(\theta_{1}, \ldots, \theta_{r}, u\right) \in[-\xi, \xi]^{r} \times[1,1+\delta]$ for any $\xi \in \mathbb{R}$ with $0<\xi<\eta_{r}$. Therefore, for $u \in[1,1+\delta]$, we can define

$$
\begin{aligned}
F_{r}\left(t_{1}, \ldots, t_{r} ; \mathbf{d}_{r} ; \mathcal{P}_{r} ; u\right) & \\
= & \sum_{N_{1}, \ldots, N_{r}=0}^{\infty} \Psi_{r}\left(d_{1}-N_{1}, \ldots, d_{r}-N_{r} ; u\right) \frac{t_{1}^{N_{1}} \cdots t_{r}^{N_{r}}}{N_{1} ! \cdots N_{r} !},
\end{aligned}
$$

which is uniformly convergent with respect to $\left(t_{1}, \ldots, t_{r}, u\right) \in \overline{\mathcal{D}}(\xi)^{r} \times$ $[1,1+\delta]$ and holomorphic for $\left(t_{1}, \ldots, t_{r}\right) \in \mathcal{D}\left(\eta_{r}\right)^{r}$ (see, for example, [7, Section 2.2]). Thus we obtain the case of $r$. This completes the proof of Theorem 1.3.

5. Some applications. First we prove the following estimates for $\Psi_{r}\left(d_{1}-N_{1}, \ldots, d_{r}-N_{r} ; u\right)$ by using the same method as in the proof of Proposition 2.3.10 in [10].

Proposition 5.1. With the same notation as in Theorem 1.3,

$$
\limsup _{N_{1}+\cdots+N_{r} \rightarrow \infty}\left\{\frac{\left|\Psi_{r}\left(d_{1}-N_{1}, \ldots, d_{r}-N_{r} ; u\right)\right|}{N_{1} ! \cdots N_{r} !}\right\}^{1 /\left(N_{1}+\cdots+N_{r}\right)} \leq \frac{1}{\eta_{r}} .
$$

Proof. Assume otherwise. Then we take $\kappa \in \mathbb{R}$ with $\kappa>1 / \eta_{r}$ such that there exist infinitely many $\left(N_{1}, \ldots, N_{r}\right) \in \mathbb{N}_{0}^{r}$ such that

$$
\frac{\left|\Psi_{r}\left(d_{1}-N_{1}, \ldots, d_{r}-N_{r} ; u\right)\right|}{N_{1} ! \cdots N_{r} !}>\kappa^{N_{1}+\cdots+N_{r}} .
$$

This means that the right-hand side of (1.10) does not converge absolutely at $(1 / \kappa, \ldots, 1 / \kappa) \in \mathcal{D}\left(\eta_{r}\right)^{r}$, which is a contradiction.

EXAMPLE 5.2. Let $\psi_{j}(s)=L\left(s ; f_{j}\right)(1 \leq j \leq r)$ as considered in Remark 3.2. Then (5.1) gives

$$
\limsup _{N_{1}+\cdots+N_{r} \rightarrow \infty}\left\{\frac{\left|L_{r}\left(d_{1}-N_{1}, \ldots, d_{r}-N_{r} ; f_{1}, \ldots, f_{r}\right)\right|}{N_{1} ! \cdots N_{r} !}\right\}^{1 /\left(N_{1}+\cdots+N_{r}\right)} \leq \frac{1}{\eta_{r}},
$$

where each $\Re d_{j}>1(1 \leq j \leq r)$ and $\eta_{r}=\min _{1 \leq k \leq r}\left\{2 \pi / 2^{r-1} m_{k}\right\}$.

Secondly we give certain multiple analogues of both Berndt's and Katsurada's formulas considered in Example 2.2. 
EXAMPLE 5.3. As in the above example, let $\psi_{j}(s)=L\left(s ; f_{j}\right)(1 \leq j \leq r)$ and define a generalization of multiple polylogarithm by

$$
\begin{aligned}
\mathcal{F}_{r}\left(t_{1}, \ldots, t_{r} ; \mathbf{d}_{r} ; f_{1}, \ldots, f_{r}\right) & \\
& =\sum_{n_{1}, \ldots, n_{r}=1}^{\infty} \frac{f_{1}\left(n_{1}\right) \cdots f_{r}\left(n_{r}\right) \prod_{j=1}^{r} e^{\left(\sum_{\mu=1}^{j} n_{\mu}\right) t_{j}}}{n_{1}^{d_{1}}\left(n_{1}+n_{2}\right)^{d_{2}} \cdots\left(n_{1}+\cdots+n_{r}\right)^{d_{r}}}
\end{aligned}
$$

for $d_{1}, \ldots, d_{r} \in \mathbb{C}$ with $\Re d_{j}>1(1 \leq j \leq r)$. Theorem 1.3 with $\psi_{j}(s)=$ $L\left(s ; f_{j}\right)(1 \leq j \leq r)$ and $u=1$ shows that $\mathcal{F}_{r}\left(t_{1}, \ldots, t_{r} ; \mathbf{d}_{r} ; f_{1}, \ldots, f_{r}\right)$ is defined and holomorphic for $\left(t_{1}, \ldots, t_{r}\right) \in \mathcal{D}\left(\eta_{r}\right)^{r}$ such that

$$
\begin{aligned}
& \mathcal{F}_{r}\left(t_{1}, \ldots, t_{r} ; \mathbf{d}_{r} ; f_{1}, \ldots, f_{r}\right) \\
& \quad=\sum_{N_{1}, \ldots, N_{r}=0}^{\infty} L_{r}\left(d_{1}-N_{1}, \ldots, d_{r}-N_{r} ; f_{1}, \ldots, f_{r}\right) \frac{t_{1}^{N_{1}} \cdots t_{r}^{N_{r}}}{N_{1} ! \cdots N_{r} !},
\end{aligned}
$$

where $\eta_{r}=\min _{1 \leq k \leq r}\left\{2 \pi / 2^{r-1} m_{k}\right\}$. Putting $t_{1}=\cdots=t_{r-1}=0$ and $t_{r}=$ $\pm i \theta$ for $\theta \in\left(-\eta_{r}, \eta_{r}\right)$ in (5.4), we have

$$
\begin{aligned}
& \sum_{n_{1}, \ldots, n_{r}=1}^{\infty} \frac{f_{1}\left(n_{1}\right) \cdots f_{r}\left(n_{r}\right) \cos \left(\left(n_{1}+\cdots+n_{r}\right) \theta\right)}{n_{1}^{d_{1}}\left(n_{1}+n_{2}\right)^{d_{2}} \cdots\left(n_{1}+\cdots+n_{r}\right)^{d_{r}}} \\
&=\sum_{N=0}^{\infty} L_{r}\left(d_{1}, \ldots, d_{r-1}, d_{r}-2 N ; f_{1}, \ldots, f_{r}\right) \frac{(i \theta)^{2 N}}{(2 N) !}
\end{aligned}
$$

REMARK 5.4. In the case $f_{j}(n)=(-1)^{n}(1 \leq j \leq r)$, the function $\mathcal{F}_{r}\left(i \theta_{1}, \ldots, i \theta_{r} ; f_{1}, \ldots, f_{r}\right)$ has recently been used to prove what is called the parity result for Euler-Zagier sums (see [15]).

\section{References}

[1] S. Akiyama and H. Ishikawa, On analytic continuation of multiple L-functions, in: Analytic Number Theory (Beijing/Kyoto, 1999), Dev. Math. 6, Kluwer, Dordrecht, 2002, 1-16.

[2] T. Arakawa and M. Kaneko, On multiple L-values, J. Math. Soc. Japan 56 (2004), 967-991.

[3] B. C. Berndt, Character analogues of the Poisson and Euler-MacLaurin summation formulas with applications, J. Number Theory 7 (1975), 413-445.

[4] J. M. Borwein, D. M. Bradley, D. J. Broadhurst and P. Lisonek, Special values of multidimensional polylogarithms, Trans. Amer. Math. Soc. 353 (2001), 907-941.

[5] K. Dilcher, Zeros of Bernoulli, generalized Bernoulli and Euler polynomials, Mem. Amer. Math. Soc. 386 (1988).

[6] A. B. Goncharov, Multiple polylogarithms, cyclotomy and modular complexes, Math. Res. Lett. 5 (1998), 497-516.

[7] L. Hörmander, An Introduction to Complex Analysis in Several Variables, 3rd ed., North-Holland, 1990. 
[8] K. Kamano, Multiple zeta values at non-positive numbers, Master Thesis, Waseda Univ., 2004 (in Japanese).

[9] M. Katsurada, Rapidly convergent series representations for $\zeta(2 n+1)$ and their

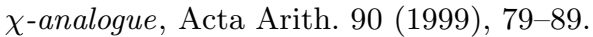

[10] S. G. Krantz, Function Theory of Several Complex Variables, Wiley, Chichester, 1982 .

[11] K. Matsumoto, Asymptotic expansions of double zeta-functions of Barnes, of Shintani, and Eisenstein series, Nagoya Math. J. 172 (2003), 59-102.

[12] - , The analytic continuation and the asymptotic behaviour of certain multiple zeta functions I, J. Number Theory 101 (2003), 223-243.

[13] - , The analytic continuation and the asymptotic behaviour of certain multiple zeta functions II, in: Analytic and Probabilistic Methods in Number Theory, in Honour of J. Kubilius (Palanga, 2001), A. Dubickas et al. (eds.), TEV, Vilnius, 2002, 188-194.

[14] K. Matsumoto and Y. Tanigawa, The analytic continuation and the order estimate of multiple Dirichlet series, J. Théor. Nombres Bordeaux 15 (2003), 267-274.

[15] H. Tsumura, Combinatorial relations for Euler-Zagier sums, Acta Arith. 111 (2004), 27-42.

[16] L. C. Washington, Introduction to Cyclotomic Fields, 2nd ed., Springer, New York, 1997.

Graduate School of Mathematics

Nagoya University

Chikusa-ku

Nagoya 464-8602, Japan

E-mail: kohjimat@math.nagoya-u.ac.jp
Department of Mathematics and Information Sciences Tokyo Metropolitan University 1-1, Minami-Ohsawa, Hachioji Tokyo 192-0397, Japan E-mail: tsumura@comp.metro-u.ac.jp

Received on 9.9.2005

and in revised form on 17.5.2006 